\title{
Akademische Bildung und die Unterscheidung von Breiten- und Elitebildung
}

\author{
Elitebildungsprogramme deutscher Hochschulen
}

\begin{abstract}
Von Manfred Stock
Zusammenfassung: Bis zum zweiten Weltkrieg galt akademische Bildung in Deutschland als Elitebildung. Danach wandelte sich ihr Charakter. Im Zuge der Hochschulexpansion wurde sie zur Breitenbildung. Vor dem Hintergrund dieser Entwicklung sind die in Deutschland seit Ende der 1990er Jahre zu registrierenden Bemühungen zu sehen, die Unterscheidung zwischen einer „Elite“und einer damit gleichsam ,gewöhnlichen“ Bildung in die akademische Bildung selbst einzuführen. Im Beitrag wird dies vor allem am Beispiel so genannter „Elitestudiengänge“ und so genannter „Intensivstudiengänge“ untersucht. Es wird den Bildungsvorstellungen nachgegangen, die diese Programme implizieren.
\end{abstract}

\section{I.}

Bis zum zweiten Weltkrieg hatte die akademische Bildung den Charakter einer Elitebildung. Im Gegensatz zur elite education, um auf eine viel zitierte Unterscheidung Martin Trows (1972) zurückzugreifen, fand die mass education jenseits der Universitäten und Hochschulen statt. Mit der Hochschulexpansion entwickelte sich die Hochschulbildung mehr und mehr zur mass education, zur Breitenbildung. Die Zahl und der Anteil derjenigen, die eine akademische Bildung erwerben, wuchsen im Weltmaßstab beständig an, mit zunehmender Beschleunigung seit den 60er Jahren des 20. Jahrhunderts (Schoefer / Meyer 2005). Allein unter den Bedingungen von Diktaturen, etwa in lateinamerikanischen und osteuropäischen Ländern, kontrahierte zeitweilig die Beteiligung an der Hochschulbildung (Reisz / Stock 2007 a).

Die Universität, die Forschung und Lehre vereint, verkörpert das Leitbild, dem sich die Einrichtungen des Hochschulbereiches dabei anzunähern suchen. Dies gilt für die USA (Lenhardt / Reisz / Stock 2008; McCormick / Zhao 2005: 53; Clark 1997: 250 ff.) und es gilt auch für Deutschland. Auch hier haben sich die Fachhochschulen bislang auf die Universitäten zu bewegt (Lenhardt 2005). Universitäten wie Fachhochschulen ermöglichen eine akademische Bildung, die sich aus der Entwicklung wissenschaftlichen Wissens speist, also in Distanz zur beruflichen Praxis gewonnen wird und nicht aus der Verallgemeinerung eines berufspraktischen Wissens hervorgeht. Sie beruht auf dem spezifischen Universalismus der Wissenschaft. Wissenschaftliches Wissen erhebt einen universellen Geltungsanspruch (Merton 1959: 552 ff), es ermöglicht universelles Lernen und zugleich universelle Kritisierbarkeit. In modernen Hochschulen werden damit Wissensbegriffe vertreten, die der Sache nach auf Inklusion orientieren. Sämtliche Personengruppen gelten als potentielle Adressaten akademischer Bildung (Stichweh 2007; Ramirez 2006). Weder Abkömmlinge bestimmter Schichten oder Klassen noch die Trägerinnen und Träger bestimmter askriptiver Merkmale gelten als ungeeignet für den Hochschulbesuch. Der spezifische Universalismus der Wissenschaft (Stock 2005: 337) verhält sich gleichsam homolog zur universellen Expansion akademischer Bildung.

Konnte Friedrich Paulsen um die Wende zum 20. Jahrhundert die „Gesamtheit der akademisch Gebildeten“ in Deutschland noch als ,eine Art geistiger Aristokratie“ beschreiben, die sich ,auf Grund der akademischen Bildung als sozial Gleichstehende“(1902: 149) anerkennen, und zwar als Angehörige einer Elite, so hat mit der Hochschulexpansion diese Beschreibung an Bedeutung verloren. 
Im Gegensatz etwa zu den USA wurde allerdings in Deutschland der Übergang der akademischen Bildung von einer Elite- zur Breitenbildung stets auch als Niedergangsgeschichte interpretiert. Zunächst galt er einigen Beobachtern als Ausdruck kultureller Entwertung. „Das ganze Elend kommt daher", so klagte Eduard Spranger schon lange vor der dramatischen Hochschulexpansion der 1960er und 1970er Jahre, „daß wir den Elitegedanken verleugnet haben. Die Masse überflutet die Rechte des Geistes“" (zitiert nach Titze 1999: 9). Zu Beginn der 1960er Jahre stieß der Deutsche Ausschuss für das Erziehungs- und Bildungswesen auf ähnliche Vorstellungen, nach denen die Einrichtungen der höheren Bildung, die den Zugang zu ,geistig führenden“ Berufen ermöglichten, jenen vorzubehalten seien, die den entsprechenden ,theoretischen Typ“ (Deutscher Ausschuß 1963: 3) der Begabung repräsentierten.

Zum Gesichtspunkt der Begabung kommt jener des „Wirtschaftsstandortes“ hinzu, den es nun, angesichts eines sich verschärfenden Wettbewerbes im globalen Maßstab, mittels Eliteuniversitäten zu sichern gelte. Die Hochschulrektorenkonferenz in der Person ihres damaligen Vizepräsidenten hat 2004 diesen Gesichtspunkt wie folgt formuliert:

„Die Bundesregierung hat in der Erkenntnis der notwendigen Sicherung des Wirtschaftsstandortes Deutschland durch die Stärkung seiner Innovationskraft die Förderung von Elite-Universitäten zum Ziel mit hoher Priorität erklärt und damit eine wichtige, längst notwendige gesellschaftliche Diskussion aus dem Hintergrund des öffentlichen Interesses in das helle Licht der öffentlichen Aufmerksamkeit gezogen “ (Kutzler 2004: 1).

Ursprünglich war die Formel von den Eliteuniversitäten im Zusammenhang mit der Exzellenzinitiative des Bundes und der Länder entstanden, in deren Mittelpunkt die Förderung von Forschungsschwerpunkten stand. Dem soll hier nicht weiter nachgegangen werden (vgl. Münch 2007, 2009; Bloch / Keller / Lottmann / Würmann 2008). Mittlerweile wird die Zuschreibung eines Elitestatus aber auch auf die Lehre ausgedehnt. Das prominenteste Beispiel hierfür ist das vom Freistaat Bayern getragene „Elitenetzwerk Bayern“, dem einundzwanzig so genannte „Elitestudiengänge“ an neun bayerischen Universitäten angehören. Hochschulpolitischen Vorgaben zufolge sollen hier ,elitäre Lehrinhalte“ (Seib 2004: 43) vermittelt werden. Daneben treten aber auch einige private Hochschulen in Deutschland in jüngster Zeit mit dem Anspruch auf, „künftige Führungseliten“ auszubilden.

\section{II.}

Die Bemühungen um die Etablierung einer Elitebildung konfrontieren die deutschen Hochschulen mit einem für sie neuen stratifizierenden Unterscheidungsschema (Kreckel 2008). Damit ist verbunden, dass die Unterscheidung von elite education und mass education erneut in das Bildungssystem eingeführt wird, nun in den Bereich der akademischen Bildung selbst. Dies impliziert die Vorstellung, dass es eine spezifische akademische Bildung elitären oder exzellenten Charakters gäbe, die der gewöhnlichen und allgemein verbreiteten akademischen Bildung überlegen sei. Damit stellt sich die Frage, wie diese Überlegenheit konstruiert wird. Oder mit anderen Worten formuliert: Wie wird der „Mehrwert“ eines als „exzellent“ oder „elitär“ geltenden Angebots akademischer Bildung gegenüber dem allgemeinen Angebot begründet? Welche Bildungsvorstellungen und -begriffe werden dabei in Anschlag gebracht? Um die Beantwortung dieser Fragen soll es im Folgenden gehen.

Geht man von der Prämisse aus, dass die akademische Bildung auf dem spezifischen Universalismus der Wissenschaft beruht, so ist es der Sache nach unmöglich, in ihrem Rahmen ein besonderes Segment unter Zugrundelegung eines hierarchischen Schemas auszuzeichnen und als ,elitär“ zu markieren. Es liegt also die Vermutung nahe, dass die entsprechenden Versuche Aporien erzeugen werden. 
Man kann sich dies zunächst an einem Beispiel aus der untergegangenen Welt des Staatssozialismus verdeutlichen. So war man noch in der DDR auf die Idee gekommen, bestimmte Bereiche eines akademischen Wissens als exklusive Bereiche eines Geheimwissens für Führungsgruppen zu reservieren. Der Marxismus-Leninismus galt als wissenschaftliche Beschreibung der Welt, auf dessen Grundlage man gesellschaftliche Gesetze erkennen und entsprechend die Gesellschaft planmäßig gestalten könne. Diese Aufgabe oblag der kommunistischen Partei. Für deren Führung gab es spezielle Parteihochschulen, die auf den Marxismus-Leninismus spezialisiert waren und entsprechende akademische Abschlüsse und Promotionen verteilten. Hier wurde ein Wissen vermittelt, das für die Parteiavantgarde reserviert war und deren führende Rolle in der Gesellschaft legitimieren und befestigen sollte. Lehrbriefe und andere Unterrichtsmaterialien waren geheim und für die Öffentlichkeit nicht zugänglich. Dies mag als eine in sich konsistente Lösung erscheinen, denn die Aporien, die einer ,,akademischen Elitebildung“ entspringen, waren hier, allerdings auf Kosten des akademischen Universalismus, ausgeschaltet. Diese „Lösung“ des Problems steht aus nahe liegenden Gründen für die gegenwärtigen Bemühungen um die Institutionalisierung einer akademischen Elitebildung nicht mehr zur Verfügung.

Gleichwohl stellt sich die Frage, wie mit der genanten Aporie in den aktuellen Elitebildungsinitiativen umgegangen wird. Diese Frage soll in zwei Schritten behandelt werden. Zunächst (III.) werden zwei allgemeine Figuren der Bearbeitung des Problems vorgestellt, die in den unterschiedlichen Bemühungen um die Institutionalisierung einer Elitebildung zutage treten. Ich beziehe das Material dabei sowohl aus hochschulpolitischen Vorgaben als auch aus den Selbstbeschreibungen, die die Hochschulen beispielsweise über Elitestudiengänge anfertigen. Sodann (IV.) werde ich mich der Diskussion in der Beratungsliteratur zuwenden, die sich mit dem Management und der Akkreditierung von Elitestudienangeboten befasst. Dies ist deswegen besonders instruktiv, weil hier Empfehlungen darüber abgegeben werden, wie die Elitebildungsangebote systematisch in das neue Studiengangssystem und die damit verknüpften formalen Standards (Stichwort: ETCS) zu integrieren sind. Dies impliziert besondere Konsistenzansprüche, vor deren Hintergrund zu erwarten ist, dass die genannten Aporien in scharfen Konturen hervortreten.

Um nicht nur auf deduktivem Wege, sondern auch kurz empirisch die Triftigkeit der Frage zu untermauern und zu zeigen, dass es unter den Geltungsbedingungen des wissenschaftlichen Universalismus keineswegs ein triviales Problem ist, den „Mehrwert“ eines elitären akademischen Bildungsangebotes gegenüber einem „gewöhnlichen“ akademischen Angebot zu begründen, möchte ich auf eine Diskussion zur Einführung der bereits erwähnten Elitestudiengänge in Bayern verweisen. Sie wurde auf dem 30. Bayerischen Hochschultag zum Thema „Braucht Deutschland Elitestudiengänge?“"geführt. Von hochschulpolitischer Seite wurde hier der Anspruch formuliert, spezielle Studiengänge ins Leben zu rufen, die „Elitemodule“ (Goppel 2004 a: 39) zur Förderung ,,der leistungswilligen und leistungsfähigen jungen Leute“ (ebd.: 33) anbieten sollen. Die „Gestaltungsgrundsätze für Elitestudiengänge“ wurden dabei wie folgt definiert: Es gehe um die „Profilierung des fachlichen Angebotes“, Internationalisierung der Studiengänge, ,,hohe Betreuungsintensität“ mit regelmäßiger Überprüfung, „Erweiterung des geistigen Horizontes und der Persönlichkeitsbildung der Studenten“, die ,,vertiefte Vermittlung von Schlüsselqualifikationen“ sowie um eine „Hinführung zu einem postgradualen Studium bzw. zu einer wissenschaftsbezogenen beruflichen Tätigkeit in verantwortlicher Position durch möglichst frühzeitige Teilhabe an der Forschung“" (Goppel 2004 b: 82).

In der Diskussion hat ein führender Vertreter der Wirtschaft zu diesen Ausführungen so Stellung genommen:

„, Wenn ich das lese, frage ich mich, wie denn sonst an den Universitäten gelehrt wird: mit Dozenten ohne Exzellenznachweis, ein Studium ohne internationale Berührungen, mit ge- 
ringer Betreuungsintensität, ohne Erweiterung des geistigen Horizontes und der Persönlichkeit, keine Hinführung zu einer beruflichen Tätigkeit in verantwortlicher Position?" (Schneevoigt 2004: 48).

Die Entgegnung verweist genau auf jene Aporien, die sich auftun, wenn im Rahmen akademischer Bildung ein besonderes Segment unter Zugrundelegung einer hierarchischen Unterscheidung als ,elitär“ ausgezeichnet werden soll. Die intendierte Unterteilung zwischen einem gewöhnlichen und einem elitären Angebot akademischer Bildung erweist sich dann schnell als ein hochschulpolitisches Manöver, das die faktische Unterversorgung der Hochschulen als Normalzustand für ein ,gewöhnliches“ akademisches Studium festzuschreiben sucht. Jene Hochschulen, die solche Studiengänge einführen, reproduzieren die Logik dieses Manövers. Entsprechend wirbt der Sprecher eines bayerischen Elitestudienganges, indem er die hervorragenden Betreuungsverhältnisse, die das Eliteprogramm auszeichnen, mit den Worten hervorhebt: „So sollte Studieren sein“ (Reif 2010).

\section{III.}

Es soll nun um zwei Figuren gehen, in denen die Aporie bearbeitet wird.

(a) In einer ersten Figur wird versucht, sie still zu stellen, indem der elitäre Charakter der Studiengänge in der Form einer Steigerungslogik beschrieben wird. So heißt es beispielsweise an der Universität Regensburg:

„Die Honors-Elitestudiengänge in den Fächern Betriebswirtschaftslehre, Volkswirtschaftslehre und Wirtschaftsinformatik sind eine spezielle Eliteförderung für die besten Absolventen der ersten Studienphase im Bachelor-Studiengang (...). Die Elitestudiengänge bewegen sich auf einem sehr anspruchsvollen wissenschaftlichen Niveau" (Fakultät Wirtschaftswissenschaften 2010).

Mit den Elitestudiengängen wird ein Übergang nach dem Schema „,anspruchvoll / sehr anspruchsvoll" in Zusammenhang gebracht. Auch in den Beschreibungen anderer Studiengänge und in den bereits zitierten Ausführungen der Hochschulpolitik zu den Elitestudiengängen in Bayern wird von dieser Figur ausführlich Gebrauch gemacht.

Hochschullehre ist auf die Präsentation und Vergegenwärtigung von Ergebnissen der wissenschaftlichen Forschung angewiesen. In diesem Zusammenhang stellt sich das Problem, die Lehre am neuesten Stand der Forschung zu orientieren. Die Unterscheidung von anspruchsvoll / sehr anspruchsvoll wird diesem Zusammenhang nicht gerecht. Ein wissenschaftliches Wissen, das im Fortgang der Forschung sich als überholt erweist, wird man kaum zugleich als wenig anspruchsvoll qualifizieren. Ebenso wäre es unangemessen, eine Lehre, die den neusten Stand der Forschung aufnimmt, als ,,sehr anspruchsvoll“" zu bezeichnen. Der neuste Stand kann morgen schon der alte sein. Auch das ,,anspruchvollste“ ökonometrische Modell schreibt zugleich jene Bedingungen fest, unter denen es sich zu Reformulierungen verpflichtet.

Die Steigerungslogik impliziert letztlich ein Bild der Wissenschaft, nach dem es ein Endziel der Wahrheitssuche gäbe, einen letzten Punkt der Wahrheit, dem man sich Schritt für Schritt annähern könne. ${ }^{1}$ Indem Eliteprogramme ihre Vorstellungen von akademischer Bildung in den Begriffen dieser Steigerungslogik konzipieren, unterstellen sie, dass man einem solchen ver-

1 Zum Perfektionsbegriff der Wahrheit vgl. Luhmann (1992: 209 ff.). 
meintlich letzten Punkt zugleich nur mit den wenigen „Besten“ oder gar „Begabtesten“ nahe kommen könnte. ${ }^{2}$

(b) Eine zweite Figur bearbeitet die Aporien dadurch, dass sie die Konstruktion eines elitären Charakters der Hochschulbildung auf außerakademische Nebenschauplätze verlagert. Angesichts der Unmöglichkeit den Mehrwert einer akademischen Elitebildung inhärent auf der Ebene der wissenschaftlichen Inhalte zu bestimmen, wird auf außerwissenschaftliche Felder ausgewichen. Eine erste Variante findet sich vor allem bei privaten Fachhochschulen, die mit dem Anspruch auftreten, zukünftige „Führungskräfte“ auszubilden, aber auch bei der bayerischen „Elite-Akademie“, einem weiteren Elitebildungsprogramm in Bayern (vgl. zur Programmatik: Durst 2004). Die Elite-Akademie soll besonders befähigte Studenten in einem dreisemestrigen studienbegleitenden Ausbildungsprogramm auf Führungsaufgaben außerhalb des Hochschulwesens vorbereiten. Dazu will sie Studenten in Kontakt bringen mit Angehörigen der etablierten Eliten. Als Lehrkräfte werden ,,erfolgreiche Führungskräfte der Wirtschaft, hochrangige Persönlichkeiten aus Medien, Politik, Stiftungen und Verbänden, namhafte Wissenschaftler und ausgezeichnete Managementtrainer" (Eliteakademie 2008) genannt. Diese Form der Elitebildung setzt nicht auf intellektuelle Unabhängigkeit von der beruflichen Praxis und will nicht die Disziplin vermitteln, die das wissenschaftliche Denken verlangt. Sie zielt vielmehr auf standesgemäße kulturelle Assimilation ${ }^{3}$ und damit verbunden auf Privilegierung beim Zugang zu Spitzenpositionen. Dieses Element der Hochschulpolitik nimmt sich aus wie ein schwacher Abglanz der Bildungsverhältnisse im Kaiserreich, die Max Weber vor Augen hatte, als er 1919 schrieb:

\begin{abstract}
„Dabei spielt, zum mindesten in Deutschland, der Wunsch mit, auf diesen Schulen in eine Couleur einzutreten, sich Schmisse ins Gesicht hauen zu lassen, satisfaktionsfähig und damit reserveoffiziersfähig zu werden und nachher im Kontor eine Vorzugschance auf die Hand der Tochter des Chefs zu haben: also sich zu assimilieren mit den Schichten der ,Gesellschaft' “ (1988: 510).
\end{abstract}

Mit dem Anspruch „künftige Führungseliten“ zu erzeugen, treten auch private Business Schools und andere private Hochschulen auf. ${ }^{4}$ Sie werben ebenfalls damit, die Studenten in Kontakt mit den führenden Vertretern der unternehmerischen Praxis zu bringen.

Eine zweite Variante bindet den elitären Charakter an die Vermittlung spezieller ,soft skills“. Exemplarisch sei aus der Beschreibung des Elitestudiengangs Softwaretechnik der Universität Augsburg zitiert:

2 Es wäre vor diesem Hintergrund ein interessantes empirisches Forschungsvorhaben zu prüfen, welche Disziplinen und Fächer auf der Grundlage welcher impliziten Weltannahmen eine besondere Affinität gegenüber der Einrichtung von Elitebildungsprogrammen unter Zugrundelegung der Steigerungslogik entwickeln. Es liegt jedenfalls die Vermutung nahe, dass wirtschaftswissenschaftliche Fächer, die dazu neigen, mit reifizierenden Modellen und entsprechenden Weltannahmen zu arbeiten (vgl. Ortlieb 2004, 2006), auch besonders affin gegenüber der Einrichtung solcher Programme sind. Dazu, dass gerade Theorien, die mit eher komplizierten mathematischen Modellen arbeiten, auch eher zu Reifikationen neigen, vgl. am Beispiel von Analysen zur Weltgesellschaft Reisz / Stock (2007 b).

3 Der schon zitierte Vertreter der Wirtschaft bemerkt zur staatlich intendierten Hochschulbildung durch Assimilation:

„,Wir können in den Unternehmen keinen dieser jungen Leute gebrauchen, die von sich glauben, den Marschallstab im Tornister zu haben, weil sie an einem angeblichen Elite-Studiengang mehr oder minder erfolgreich teilgenommen haben. Wir brauchen hochqualifizierte akademisch ausgebildete Leute, die bereit sind, im Unternehmen unten anzufangen “ (Schneevoigt 2004: 48).

4 Der Spender des Stiftungsvermögens der privaten Bucerius Law School in Hamburg hat sein Verständnis von Hochschulbildung einmal in den folgenden Worten zusammengefasst:

,Wir haben in abenteuerlichen Reformen unser Hochschulsystem statt auf Elite und Wettbewerb auf breiteste Masse umgestellt" (Zitiert nach Ankenbrand 2008: 16). 
"Highest value is put on an individual and intensive support of the students in the context of the elite program. (...) Mentors are leading decision makers of the affiliated industrial partners as well as the professors of the elite program. The education in the elite program should enable the graduates to adopt leading positions in industry and research. The ability to work in a team, competences as a leader and knowledge in rhetoric are basic requirements for this. The elite program is accompanied by education in those soft skills" (Elitestudiengang Softwaretechnik 2010).

Die so genannten soft skills werden dabei zum Gegenstand spezieller Lehrveranstaltungen, die jenseits des genuin wissenschaftlichen Programms liegen.

Da in materialer Hinsicht die Überlegenheit des akademischen Programms nicht unter Berufung auf Wissenschaftlichkeitsstandards abgeleitet werden kann, werden außerwissenschaftliche soft skills ins Feld geführt. Häufig wird deren Erwerb auch in Zusammenhang gebracht mit der Pflege spezieller Vergemeinschaftungsinstitutionen. ${ }^{5}$

IV.

Mittlerweile wurden in Deutschland akademische Studienprogramme mit elitärem Anspruch in zwei Formen institutionalisiert, in der Form des Elitestudiengangs und in der Form des Intensivstudiengangs. Beide Formen haben sich fest etabliert. Sie sind formell dem European Credit Transfer System (ECTS) unterworfen. Allerdings bringen beide Formen auch spezifische Probleme mit sich, wenn es darum geht, sie systematisch mit den formalen Standards der Bologna-Studiengangsstruktur in Übereinstimmung zu bringen. Ein Blick darauf gestattet die Beratungsliteratur, die sich mit dem Management, der Akkreditierung und den formalen Prozeduren der Anrechnung von Studienleistungen nach dem ECTS befasst. Im Lichte dieser Bewertungsstandards tritt der Geist der Eliteprogramme deswegen besonders prägnant hervor, weil die Frage nach dem Mehrwert eines elitären akademischen Bildungsangebotes unter systematischen Gesichtspunkten zu beantworten versucht wird. Ich konzentriere die Analyse auf Vorgaben, die im „Handbuch Qualität in Studium und Lehre: Evaluation nutzen - Akkreditierung sichern - Profil schärfen!“ (Benz / Kohler / Landfried 2009) enthalten sind. Es handelt sich dabei um ein beständig aktualisiertes Kompendium paradigmatischen Charakters, das die anwendungsorientierte Hochschulforschung für die Praxis als Leitfaden bereithält.

(a) Intensivstudiengänge

Die Form des Intensivstudiengangs wurde in Niedersachsen eingeführt. Das Niedersächsische Ministerium für Wissenschaft und Kultur wirbt für seine Intensivstudiengänge mit den Worten:

„Hier können Sie richtig Zeit sparen - durchschnittlich zwei bis vier Semester. Aufwand, der sich lohnt. Wer besonders begabt und belastbar ist und sein Ziel unbeirrt verfolgen will, ist hier richtig " (Hochschulen in Niedersachsen 2008: 1). ${ }^{6}$

Im genannten Handbuch werden diese Studiengänge unter dem Gesichtspunkt der ECTS-Logik wie folgt interpretiert:

5 Es ist hier nicht der Ort, um der bunten Vielfalt im Detail nachzugehen, in der die Elitebildung auf diesem Feld sich entwickelt. Das Spektrum reicht von der Teilnahme am „Hochseilgarten Honors Elitecup I und II“ und am „McKinsey Cocktailmixkurs“ (Wirtschaftswissenschaften Uni Regensburg, vgl. http://www-honors.uni-regensburg.de/honors/index.php?option=com_pics\&Itemid=94; Zugriff Februar 2010) bis hin zu „Recruiting Messen“ und „Gala Dinners“ mit Führungspersönlichkeiten aus Wirtschaft und Politik an der European Business School (EBS) Schloss Reichartshausen (vgl. dazu die instruktiven Beschreibungen und Analysen in Bloch (2009: $188 \mathrm{ff}$ ); zum genannten Beispiel S. 227).

6 Der niedersächsische Wissenschaftsminister fügt hinzu: „,Das ist kein verkürztes Schnellstudium, sondern ein Angebot für eine Elite, die besonders leistungsfähig und -bereit ist“ (Oppermann 2010). 
„, Intensivstudiengänge sind solche Studiengänge, in denen auf der Grundlage einer erhöhten Arbeitszeit in demselben Zeitraum mehr ECTS-Punkte vergeben werden als in normalen Studiengängen (...) Charakteristisch ist also die Erhöhung der Workload pro Semester bzw. Jahr im Vergleich zum Regelstudienprogramm, (...) wobei das angestrebte Qualifikationsniveau (...) im Vergleich zum Regelfall nicht verändert wird. Das bestimmende Leitmotiv lautet also: mehr Arbeit pro Tag, Monat und Jahr" (Leinweber / Mayer-Lantemann 2009: 4).

Demnach geht es also in Intensivstudiengängen schlicht darum, in einem technischen Sinne die Intensität zu steigern.

Es drängt sich förmlich auf, dies in lockerer Anlehnung an Kategorien der Marxschen Mehrwerttheorie zu beschreiben. Dies ist deswegen nicht abwegig, weil es in den Begriffen des ECTS nicht auf das Studium konkreter Inhalte ankommt. Mit Marx' Unterscheidung von konkreter und abstrakter Arbeit könnte man sagen: Das Studium wird gegenüber seinem besonderen Inhalt als gleichgültig betrachtet, es geht allein um die Verrichtung einer abstrakten „workload“, um die bloße Verausgabung von „Hirn und Nerven“.7 Das Studium wird auf eine abstrakte Tätigkeit reduziert, die sich gleichsam in einen Quasi-Tauschwert ummünzt, in eine Anzahl von ECTS-Punkten. Im Falle der Intensivstudiengänge wird dabei der Mehrwert des Eliteprogramms nach dem Produktionsschema des absoluten Mehrwertes konstruiert. Der Studierende erreicht ein ECTS-Punktäquivalent in kürzerer Zeit durch die Ausdehnung der wie es heißt - „Lernzeit“ im Studienjahr. Um das erreichen zu können, bedarf es, so heißt es in einer Festlegung des Akkreditierungsrates, ,besonderer studienorganisatorischer Maßnahmen“ (zitiert nach: Leinweber / Mayer-Lantemann 2009: 19), nämlich besserer Studienbedingungen und finanzieller Zuwendungen, damit ein größerer Teil der Lebenszeit auch tatsächlich als Lernzeit verausgabt werden kann. Dies bedeutet eine Ausdehnung der Vorlesungszeiten pro Semester sowie die Abschaffung der Semesterferien. Diese Maßnahmen erscheinen als Mittel, damit das Studienjahr intensiver genutzt werden kann. In Intensivstudiengängen sollen

7 Als Verausgabung menschlicher Arbeitkraft, die besonderen Zwecken folgt, als Erzeugung von Gebrauchswerten, so die Marxsche Unterscheidung, ist jede Arbeit konkrete Arbeit. Als tauschwertbildende Arbeit wird von ihrem materialen Inhalt abstrahiert. Sie ist allein Verausgabung menschlicher Arbeitkraft, „Verausgabung von menschlichem Hirn, Muskel, Nerv, Hand usw.“ (Marx 1983 a: 62), das heißt abstrakte Arbeit. Verschiedene Gebrauchswerte werden durch verschiedene Qualitäten konkreter Arbeit produziert, als Tauschwerte enthalten sie die gleiche Qualität: menschliche Arbeit. Die Größe des Wertes einer Ware wird durch das Quantum der in ihr enthaltenen abstrakten menschlichen Arbeit bestimmt. Dies verhält sich analog zu „,credit points“ und „,workloads“. Marx hatte die Herstellung dieser Abstraktheit der Arbeit mit dem Modernisierungsprozess der bürgerlichen Gesellschaft in Zusammenhang gebracht:

„,Die Gleichgültigkeit gegen die bestimmte Arbeit entspricht einer Gesellschaftsform, worin die Individuen mit Leichtigkeit aus einer Arbeit in die andre übergehen und die bestimmte Art der Arbeit ihnen zufällig, daher gleichgültig ist. Die Arbeit ist hier nicht nur in der Kategorie, sondern in der Wirklichkeit als Mittel zum Schaffen des Reichtums überhaupt geworden und hat aufgehört, als Bestimmung mit den Individuen in einer Besonderheit verwachsen zu sein. Ein solcher Zustand ist am entwickeltsten in der modernsten Daseinsform der bürgerlichen Gesellschaft - den Vereinigten Staaten. Hier also wird die Abstraktion der Kategorie 'Arbeit', 'Arbeit überhaupt'... erst praktisch wahr " (Marx 1983 b: 39). Die besondere Fertigkeit der Arbeit werde mit der Entwicklung des Kapitalismus „rein abstrakte Tätigkeit, rein mechanische, daher gleichgültige, gegen ihre besondre Form indifferente Tätigkeit" (Marx 1983 b: 218 f). Der Doppelcharakter der warenproduzierenden Arbeit wird so tendenziell mit fortschreitender Entwicklung des Kapitalismus aufgehoben. Es ist hier nicht der Platz, um der Frage nachzugehen, ob in der zeitgenössischen Phase der Moderne die „Kopf“-Arbeit im Zusammenhang mit der Umstellung vom Buchdruck auf das Verbreitungsmedium PC (vgl. Baecker 2007) einer ähnlichen Entwicklung unterworfen ist. Solche Marken wie „employability“, „ECTS“, „Modularisierung“, „workload“ wären dann als gleichsinnige Bestandteile einer übergreifenden Transformation zu interpretieren. 
auf diese Weise bis zu 75 Punkte pro Jahr erreicht werden (Akkreditierungsrat 2006 und 2009). Im Normalstudiengang sind es 60; dem entsprechen 1800 Stunden. Im Handbuch heißt es dazu:

„Eine damit verbundene Belastung von 2250 Zeitstunden im Jahr, verbunden mit 43,3 Zeitstunden in der Woche bei Wegfall jeglicher Urlaubszeiten (...) hält der Akkreditierungsrat noch für vertretbar" (Leinweber/Mayer-Lantemann 2009: 20).

Der Form des Intensivstudienganges entspricht ein Leitbild vom Studierenden, nach dem dieser sich selbst als Gegenstand einer technischen Rationalisierung, quasi als zu rationalisierender Mechanismus begreift, der seine Intensität zu steigern hat. ${ }^{8}$ Die formale Durchrationalisierung des Studiums wird mit den Intensivstudiengängen auf die Spitze getrieben. Es ist diese Spitzenposition, die als elitär konnotiert wird. Redete Marx noch mit Blick auf die Mehrwertproduktion von der reellen Subsumtion der proletarischen Handarbeit unter die losgelassene Maschinerie kapitalistischer Rationalisierung, so geht es jetzt im Studium um eine reelle Subsumtion der Lebenszeit unter die Zeit eines technisch durchrationalisierten Lernens. Wer eine solche Belastung auszuhalten bereit ist, darf sich zur Elite gehörig fühlen. Unten werden noch Anhaltspunkte dafür beigebracht, dass dies keine polemische Überspitzung ist, sondern bereits auch in entsprechenden Habitusformationen zur realen Existenz gelangt. Jedenfalls ist in den vorfindlichen Selbstbeschreibungen zu Eliteprogrammen nicht davon die Rede, dass es in der akademischen Bildung für den Einzelnen darauf ankomme, in der Auseinandersetzung mit einem wissenschaftlichen Gegenstand mehr als nur ein technisches Verhältnis zu sich selbst zu gewinnen.

(b) Elitestudiengänge

Schaut man sich die ECTS-Punktezuordnung beispielsweise für den oben bereits erwähnten Honors-BWL-Studiengang an, so trifft man auf die Regelung, dass neben den Modulen des regulären Studienganges im Elitestudiengang ein zusätzliches „Honors“-Modul absolviert werden muss, für das 20 Punkte angerechnet werden (Studienkonzept 2007).

Aufschlussreich sind auch hier die Betrachtungen, die im Handbuch zur Punktebewertung von Elitestudiengängen angestellt werden:

In einem Elitestudiengang, so die Logik,

„, wird mit derselben Workload pro Jahr ein höheres Qualifikationsniveau als das der dieser Workload im ,Normalfall' jeweils zugeordneten Qualifikationsstufe [erreicht]) (...) Das bestimmende Leitmotiv lautet hier: Mehr Lerneffekt je erbrachtem Arbeitseinsatz. (...) Wichtige persönliche Faktoren zur Bewältigung einer hohen Arbeitsbelastung - wie sie in ,Intensivstudiengängen' vorgesehen sind - sind Fleiß, Motivation und erhöhte Belastbarkeit. (...) In Elitestudiengängen, in denen schneller im Sinne von effektiver gelernt werden muss, kommt es dagegen eher auf individuelle Begabungen wie ein schnelles Auffassungsvermögen an " (Leinweber / Mayer-Lantemann 2009: 5).

8 Diese Logik einer versachlichenden Selbstinstrumentalisierung bringt die Werbebroschüre des Niedersächsischen Ministeriums für Wissenschaft und Kultur mit dem Titel „Kurz und gut. Intensivstudiengänge in Niedersachsen “ (2002) auf den Punkt. So heißt es über den Intensivstudiengang Physik an der TU Braunschweig „Turbo-Diplom für Ungeduldige“:

„Man kann auch schneller zur Sache kommen: Nur vier Jahre braucht man im Intensivprogramm der TU Braunschweig, um Diplom-Physikerin bzw. -Physiker zu werden, allerdings bei den gleichen Anforderungen wie im Normalstudium. Damit liegen die Programm-Absolventinnen und-Absolventen auf dem Arbeitsmarkt an der Pole-Position. Einsteigen, bitte! Welcher Nationalität Sie sind, ist egal, denn der Intensivstudiengang richtet sich an deutsche und ausländische Studierende. Hauptsache, Sie können die allgemeine Hochschulreife oder Vergleichbares vorweisen. Alles, was sie sonst noch überwinden müssen, ist ein Numerus clausus " (Niedersächsisches Ministerium 2002: 6). 
Damit effizienter ${ }^{9}$ gelernt werden könne, so folgt daraus, muss bei der Zulassung zum Elitestudiengang eine Selektion nach Begabung erfolgen. Ferner, so empfiehlt das Handbuch, seien spezielle Vorkehrungen zu treffen, ,,durch die die Studierenden, wirkungsvoller je Zeiteinheit' lernen“, vor allem gehe es dabei um eine ,überdurchschnittliche Qualität der Stoffvermittlung“ (ebd.).

Im Fall der Elitestudiengänge könnte man, um die Analogie zur Mehrwertproduktion fortzusetzen, davon sprechen, dass das Programm nach dem Schema der Produktion des relativen Mehrwerts konstruiert ist. Das Konzept geht von der Prämisse aus, dass die Studenten in der Lage seien, in einer kürzeren Lernzeit das normale Lernziel zu erreichen. Sie könnten daher bei gegebener Studiendauer zusätzliche Lernziele realisieren. Folgte man dieser Prämisse, so ergäben sich allerdings in der Sicht der Handbuchautoren Probleme bei der Bewertung der Studiengänge nach dem ECTS, die eine formal korrekte Akkreditierung der Elitestudiengänge erschwerten. ${ }^{10}$

Diesem Schluss liegt in letzter Konsequenz der folgende Gedankengang zu Grunde: Der Vergleichsmaßstab des ECTS sei den Elitestudiengängen nicht gewachsen, weil er ausschließlich auf bloßen Zeiteinheiten aufbaue, in denen gleichsam die normalbegabten Durchschnittsstudenten lernten. Um ein auf,,workloads“abstellendes Bewertungsinstrumentarium einsetzen zu können, müsse man gewissermaßen den Punkt ermitteln, an dem ein Elitestudent das normale Lernziel erreicht hat, um dann die Dauer der gleichsam Surplusstudienzeit bestimmen zu können, in der er den Vorteil seiner überdurchschnittlichen Begabung einfahre.

Bislang steht eine Entscheidung des Akkreditierungsrates bezüglich der Akkreditierung von Elitestudiengängen noch aus. Man darf gespannt sein, auf welche Weise das ETCS mit der Produktionslogik eines relativen Mehrwertes zusammengebracht werden wird.

Dieses Problem soll hier aber nicht weiter interessieren, es dient uns nur als Indikator für die Semantik, in der eine „elitäre“ akademische Bildung begründet und verhandelt wird. Um den Aporien zu begegnen, die mit der Wiedereinführung der Unterscheidung von elite- und mass education in die akademische Bildung erzeugt werden, wird eine Semantik bemüht, die zum einen ökonomistisch-technischen Charakters ist. Der Mehrwert eines elitären Programms wird im Rückgriff auf ein Schema begründet, das Marx mit Blick auf den kapitalistischen Arbeits- und Verwertungsprozess als jenes der Produktion des relativen und des absoluten Mehrwertes kritisiert hatte. Die implizite Logik dieses Produktionsschemas wird nun im Sinne eines institutionenökonomischen Paradigmas als triftig unterstellt, um akademische Bildungsprozesse zu beschreiben. Es wird nicht mehr jene hergebrachte ständisch-traditionale Semantik bemüht, die Max Weber dazu veranlasste, von einer Geistesaristokratie zu sprechen, oder Friedrich Paulsen von einem akademischen Amtsadel. Diese Semantik stellte auf einen ständischen Charakter akademischer Elitebildung ab. Zum anderen kehrt aber dieses ständische Moment auch im Begabungsbegriff zurück. Die Studierenden gelten in einem quasi-ständi-

9 Folgt man dem gängigen betriebswirtschaftlichen Vokabular, so muss es im vorangegangenen Zitat statt „effektiver“ eigentlich „effizienter“ heißen.

10 So wird die herrschende Praxis, dass einfach für Eliteprogramme mehr ECTS-Punkte vergeben werden als für gewöhnliche Studienprogramme, im Handbuch mit den Worten kritisiert: Es wird nicht deutlich,

„ob die erhöhte Anzahl an ECTS-Punkten auf einer erhöhten Arbeitsbelastung vor dem Hintergrund unter anderem einer angenommenen erhöhten Belastbarkeit oder ohne eine Arbeitszeiterhöhung unter anderem aufgrund der Annahme vergeben wird, die Studierenden ereichten das Qualifikationsniveau aufgrund einer besonders guten Auffassungsgabe bzw. besonderen Talents schneller " (Leinweber / Mayer-Lantemann 2009: 17).

Mit anderen Worten: Man könne nicht unterscheiden, ob der Mehrwert des Eliteprogramms nach der Logik des absoluten (Intensivstudiengänge) oder des relativen Mehrwertes (Elitestudiengänge) eingefahren werde. 
schen Sinne als von Natur aus festgelegt im Hinblick - ich spitze zu - auf ihren Wirkungsgrad, vorgegebene Lernziele zu erreichen.

In beiden Hinsichten werden sie als Trivialmaschinen behandelt, die nach berechenbaren Regeln und nach festgelegten zeitlichen Parametern ,inputs“ in ,outputs“ verwandeln. In den Elitebildungsprogrammen funktionieren sie, so die Vorstellung, schlicht effizienter oder effektiver.

Weil es angesichts des wissenschaftlichen Universalismus unmöglich ist, material eine Unterscheidung zwischen „elitär“ und ,gewöhnlich“ in die akademische Bildung einzuführen, werden Schemata technischer Rationalisierung bemüht, um diese Differenz zu begründen. Dabei wird auf den Bereich der akademischen Bildung der Modus schulischen Lernens übertragen, welcher darauf abzielt, kanonisierte Wissensbestände zu vermitteln.

V.

Da das Handeln in Forschung und Lehre, also im Kernbereich der Hochschulen, unmittelbar den Normen des wissenschaftlichen Universalismus unterworfen ist, wäre zu erwarten, dass die Hochschulen gegenüber dem Ansinnen, Elitebildungsprogramme zu installieren, Widerstände entwickeln müssten. Die im Abschnitt III aufgeführten Befunde deuten darauf hin, dass dies nicht der Fall ist. Die Universitäten haben Eliteprogramme eingeführt, und bis auf die Ebene der Fachbereiche hinein haben sie Selbstbeschreibungen entwickelt, die jene Programme normativ stützen und inhaltlich zu begründen suchen. Zunächst könnte die Vermutung nahe liegen, dass diese Situation mit einem Ritualismus einhergeht: Um in den Genuss der nicht unerheblichen zusätzlichen Finanzmitte ${ }^{11}$ zu gelangen, die der Staat für Eliteprogramme bereitstellt, werden diese formell eingerichtet, entsprechende Selbstbeschreibungen als „talk“12 erzeugt, und die zusätzlichen Selektionsmöglichkeiten werden gern als Gelegenheit willkommen geheißen, sich endlich allein mit jenen wenigen Studenten befassen zu können, die für eine forschungsnahe Lehre tatsächlich als geeignet erscheinen. Solche Institutionen wie der „Hochseilgarten Honors Elitecup I und II“ oder auch der „McKinsey Cocktailmixkurs“, die die Wirtschaftswissenschaften an der Universität Regensburg als Bestandteil ihrer Eliteprogramme eingerichtet haben, ${ }^{13}$ erscheinen dann eher als empirische Belege für eine symbolische Überproduktion, die einer Logik von „talk“ folgt, aber von der Ebene der ,action“ entkoppelt, also weitgehend folgenlos im Hinblick auf die faktischen Interaktionen in Lehre und Forschung bleibt. Man hält im Kern an den Prinzipien eines spezifisch wissenschaftlichen Universalismus fest und bedient ritualistisch die Elitesemantik als symbolisches Zugeständnis an die überzogenen Erwartungen der Hochschulpolitik. Eine solche Vermutung würde die neoinstitutionalistische Organisationssoziologie (Meyer / Rowan 1977) nahe legen.

Aber auch Riten und Mythen müssen reproduziert und aufrechterhalten werden. Und die beispielhaft aufgeführten Institutionen der Elitebildung konterkarieren ganz offensichtlich die Professionsnormen, die in Universitäten im Allgemeinen als geltend vorausgesetzt werden (vgl. Parsons / Platt 1973; Oevermann 2005). Auch ein ritualistischer Umgang mit diesen Institutionen würde daran nichts ändern. Es ist daher zu vermuten, dass jene Hochschulen und

11 Auf der Webseite des Elitenetzwerkes Bayern heißt es dazu: „Staatsregierung und Landtag stellen 223 hochwertige Stellen für Elitestudiengänge und Doktorandenkollegs im Elitenetzwerk Bayern bereit. Zusätzlich wurden 14 Mio. Euro als Anschubfinanzierung zur Verfügung gestellt" (Vgl.: https://www.elitenetzwerk.bayern.de/ 135.0.html\#c4701; Zugriff: Februar 2010).

12 Ich greife hier auf die Unterscheidung von „talk“, „,decision-making“ und ,action“ (Brunsson 1982, 1989, 1995) zurück. Zur Anwendung dieses Ansatzes auf die Analyse von Hochschulorganisationen im Falle der Einführung des New Public Management vgl. Stock (2004).

13 Vgl. Fußnote 5. 
Fachbereiche sich die Elitesemantik zu Eigen machen, in denen homologe Orientierungen ohnehin bereits an Bedeutung gewonnen haben.

Für den Bereich der öffentlichen Hochschulen gibt es meines Wissens dazu keine empirischen Untersuchungen. Erste Fallstudien liegen allerdings für private Hochschulen vor, die sich selbst als Einrichtungen einer Elitebildung präsentieren. Sie legen den Schluss nahe, dass jener Geist, der der Elitesemantik entspricht, auch jene normativen und kognitiven Standards nicht unberührt lässt, die der Lehre faktisch zu Grunde gelegt werden. Mit Blick auf die Beratungsliteratur wurde gezeigt, dass die Aporien, die sich mit der Einführung der Unterscheidung von elite und mass education in die akademische Bildung verbinden, im ökonomistischtechnischen Schema der absoluten und relativen Mehrwertproduktion zum Schweigen gebracht werden. Die Semantik dieses Schemas konzipiert die Studierenden als Virtuosen funktionstüchtiger Selbstinstrumentalisierung. Die entsprechenden Fähigkeiten gelten als elitär. Erste Untersuchungen zum Studium an privaten Hochschulen, die sich als hoch selektiv präsentieren und einem Elitebildungsauftrag verpflichtet fühlen, gelangen zu Befunden, die genau einem solchen Rollenbild entsprechen (vgl. Bloch 2009: $181 \mathrm{ff}$ ). Ich zitiere zur Illustration zwei Beispiele aus den Interviewprotokollen:

Markus: „Ich glaube, die beste Eigenschaft, die Eigenschaft, die auch die WHUler haben, die auch eher schwach sind, also das ist unbedingter Wille und Bereitschaft, Leistungsbereitschaft. Und unbedingt ist nicht unbedingt positiv anzusehen. Also das ist schon, das ist eine Sache, die man unbedingt mitbringen muss. Na klar, man muss smart sein, aber BWL ist nicht Astrophysik, ja. Also das ist schon alles verständlich gemacht, das kann schon jeder verstehen. Die Frage ist halt, in wie kurzer Zeit du das lernen musst. Das ist so eine Sache. Man muss smart sein im Sinne von dass man in kurzer Zeit viel lernen kann. Also Leistungsbereitschaft, Leidensfähigkeit ein bisschen " (zitiert nach Bloch 2009: 193).

Nina: „Also das Krasse ist: der Bachelor hier (an der EBS - d.A.), wenn wir das jetzt vergleichen mit einem BWL-Studium an einer öffentlichen Uni im Diplom, machen wir halt dasselbe oder noch mehr in unserem Bachelorstudiengang. Was halt damit zusammenhängt, dass vom Elitestatus her die Leute geprüft werden, ob sie mit dieser krassen Belastung auskommen " (zitiert nach Bloch 2009: 187).

Selbstreflexion in der Auseinandersetzung mit einem wissenschaftlichen Gegenstand wäre das Gegenteil jenes blinden Fleißes, zu dem die hier zitierte Gestalt der Hochschulbildung sich entschlossen hat. Sie widerspricht einer akademischen Bildung, denn sie wird von vorn herein bestimmt von der Aneignung eines bereits Vorgegebenen und als unhinterfragbar Vorausgesetzten, in dem der Lernende selbst, sein Urteil, gleichsam abwesend ist. Dem spezifisch wissenschaftlichen Universalismus entspricht die Forderung, nichts blind zu akzeptieren, ihm widerspricht die Bevormundung durch heteronome Dogmen. Das Studium, von dem im Zitat die Rede ist, ist hingegen zu einer Gestalt der Heteronomie geworden, die darin dem Einzelnen ein hohes Maß an technischer Verfügung über sich selbst abpresst. Der Anspruch, der sich mit einer akademischen Bildung verbindet, wird in diesem Fall in der Realität des Eliteprogramms auch faktisch aufgegeben.

\section{VI.}

Mit der Einführung der Unterscheidung von Elite- und Breitenbildung in die akademische Bildung, so konnte gezeigt werden, wird den entsprechenden Elitebildungsprogrammen ein technischer Charakter zugeschrieben. Als kollektive Größe der Sozialstruktur erscheint „Elite“ damit zugleich in einem neuen Licht. Bei Adorno findet sich ein Hinweis auf eine soziale Konstellation, in der sich eine Elite selbst dementiert: Indem sich eine Elite selbst als solche beschreibe, verwandele sie sich in das Gegenteil dessen, was sie zu sein beanspruche. „Elite“, 
so lesen wir bei ihm, „mag man in Gottes Namen sein; niemals darf man als solche sich fühlen“ (1963: 165). Adorno setzte noch voraus, dass sich Elite auf Charisma gründet.

Die untersuchten Programme der Elitebildung, so lässt sich in Anlehnung an Max Weber resümieren, verhelfen der Technik zum Sieg über das Charisma. Indem sie die Unterscheidung von Elite- und Massenbildung in die akademische Bildung einführen, tragen sie zugleich zur Entcharismatisierung der Vorstellung über eine Elite bei, deren Stellung sie zu befestigen suchen.

\section{Literatur:}

Adorno, Theodor W. (1963): Meinung Wahn Gesellschaft, in: Eingriffe. Neun kritische Modelle. Frankfurt / Main, S. 147-172.

Akkreditierungsrat (2006): Vergabe von ETCS-Punkten in Intensivstudiengängen. Drs. AR 24/2006.

Akkreditierungsrat (2009): Regeln des Akkreditierungsrates für die Akkreditierung von Studiengängen und für die Systemakkreditierung. Drs. AR 93/2009.

Ankenbrand, Hendrik (2008): Hamburger Eliten-Leuchtturm, in: Frankfurter Allgemeine Zeitung vom 27.9.2008.

Baecker, Dirk (2007): Studien zur nächsten Gesellschaft. Frankfurt / Main.

Bloch, Roland (2009): Flexible Studierende? Studienreform und studentische Praxis. Leipzig.

Bloch, Roland / Andreas Keller / Andre Lottmann / Carsten Würmann (2008): Making Excellence. Grundlagen, Praxis und Konsequenzen. Bielefeld.

Brunsson, Nils (1982): The Irrationality of Action and Action Rationality: Decisions, Ideologies and Organizational Actions, in: Journal of Management Studies 19, S. 29-44.

Brunsson, Nils (1989): The Organization of Hypocrisy. New York.

Brunsson, Nils (1995): Ideas and Actions: Justification and Hypocrisy as Alternatives to Control, in: Research in the Sociology of Organisation 13, S. 211-235.

Clark, Burton R. (1997): The Modern Integration of Research Activities with Teaching and Learning. In: Journal of Higher Education 68, S. 241-255.

Deutscher Ausschuss für das Erziehungs- und Bildungswesen (1963): Rahmenplan zur Umgestaltung und Vereinheitlichung des allgemeinbildenden öffentlichen Schulwesens. Stuttgart.

Durst, Franz (2004): Die Bayerische Eliteakademie. Ziele und Aufgaben, in: Beiträge zur Hochschulforschung 26, H. 3, S. 32-37.

Eliteakademie (2008): Vgl.: http://www.eliteakademie.de [Zugriff am 1.9.2008].

Elitestudiengang Sotwaretechnik (2010): Vgl.: https://www.elitenetzwerk.bayern.de/61.0.html und http:// www.uni-augsburg.de/elite/se/curriculum/ [Zugriff am 1.2.2010].

Fakultät Wirtschaftswissenschaften (2010): Honors-Elitestudiengänge. Vgl.: http://www-wiwi.uni-regensburg.de/Studium/Honors/index.html.de [Zugriff am 1.2.2010].

Goppel, Thomas (2004 a): Das Bayerische Elitenetzwerk: ein Modell, in: Politische Studien, Heft 398, 55 Jg., S. 33-39.

Goppel, Thomas (2004 b): Woran denkt die Politik beim Ruf nach Elitestudiengängen? In: Beiträge zur Hochschulforschung 26, H. 3, S. 78-87.

Hochschulen in Niedersachsen (2008): Vgl.: http://nds.studieren.niedersachsen.de/de/sin/ studiengaenge.php [Zugriff am 1.2.2010].

Kreckel, Reinhard (2008): Zwischen universeller Inklusion und neuer Exklusivität. Institutionelle Differenzierung und Karrieremuster im akademischen Feld: Deutschland im internationalen Vergleich, in: Barbara Kehm (Hg.): Hochschule im Wandel. Festschrift für Ulrich Teichler. Frankfurt a.M. / New York, S. 181-194. 
Kutzler, Kurt (2004): Begrüßungsrede zur Jahresversammlung der Hochschulrektorenkonferenz in Berlin 2004. Vgl.: http://www.hrk.de/de/download/dateien/Begruessungsrede_Kurt_Kutzler.pdf [Zugriff am 1.1.2010].

Leinweber/Mayer-Lantemann (2009): Im Spagat zwischen den Anforderungen ECTS-basierter Vergleichbarkeit und programmatischer Profilbildung. Der Fall der „Intensiv- und Elitestudiengänge“, in: Winfried Benz / Jürgen Kohler / Klaus Landfried (Hrsg.), Evaluation nutzen - Akkreditierung sichern - Profil schärfen! Teil F 5.2. Berlin, S. 1-24.

Lenhardt, Gero (2005): Hochschulen in Deutschland und in den USA. Wiesbaden.

Lenhardt, Gero / Robert D. Reisz / Manfred Stock (2008): Amerikanische „Elitehochschulen“ - selective colleges and major research universities, in: Zeitschrift für Erziehungswissenschaft 11, S. 559-576.

Luhmann, Niklas (1992): Die Wissenschaft der Gesellschaft. Frankfurt / Main.

Marx, Karl (1983 a): Das Kapital. Bd. 1. In: Karl Marx / Friedrich Engels: Werke, Bd. 23. Berlin.

Marx, Karl (1983 b): Grundrisse der Kritik der Politischen Ökonomie. In: Karl Marx / Friedrich Engels: Werke, Bd. 42. Berlin.

McCormick, Alexander C. / Chun-Mei Zhao (2005): Rethinking and Reframing the Carnegie Classification, in: Change, September/October, S. 51-57.

Merton, Robert K. (1959): Social Theory and Social Structure. Glencoe.

Meyer, John W. / Brian Rown (1977): Institutionalized Organizations: Formal Structure as Myth and Ceremony, in: American Journal of Sociology 83, S. 340-363.

Münch, Richard (2007): Die akademische Elite. Zur sozialen Konstruktion wissenschaftlicher Exzellenz. Frankfurt / Main.

Münch, Richard (2009): Globale Eliten, lokale Autoritäten. Bildung und Wissenschaft unter dem Regime von PISA, McKinsey\&Co. Frankfurt / Main.

Niedersächsisches Ministerium für Wissenschaft und Kultur (2002): Kurz und gut. Intensivstudiengänge in Niedersachsen. Hannover.

Oevermann, Ulrich (2005): Wissenschaft als Beruf. Die Professionalisierung wissenschaftlichen Handelns und die gegenwärtige Universitätsentwicklung. In: Manfred Stock / Andreas Wernet (Hrsg.), Hochschule und Professionen, in: Die Hochschule. Journal für Wissenschaft und Bildung 14, H.1, S. 15-51.

Oppermann, Thomas (2010): Interview, in: Hildesheimer Allgemeine Zeitung vom 6.6.2001, vgl.: http:// www.uni-hildesheim.de/de/17383.htm [Zugriff 2.2.2010].

Ortlieb, Claus Peter (2004): Methodische Probleme und methodische Fehler der mathematischen Modellierung in der Volkswirtschaftslehre, in: Hamburger Beiträge zur Modellierung und Simulation, H. 18, Januar, 1-22.

Ortlieb, Claus Peter (2006): Mathematisierte Scharlatanerie, in: Thomas Dürmeier / Tanja v. Egan-Krieger / Helge Peukert (Hrsg.): Die Scheuklappen der Wirtschaftswissenschaft. Postautistische Ökonomik für eine pluralistische Wirtschaftslehre. Marburg, S. 55-62.

Parsons, Talcott / Gerald M. Platt (1973): The American University. Cambridge.

Paulsen, Friedrich (1902): Die deutschen Universitäten und das Universitätsstudium. Berlin.

Ramirez, Francisco O. (2006): The Rationalization of Universities, in: Marie-Laure Djelic / Kerstin ShalinAndersson (Hrsg.): Transnational Governance: Institutional Dynamics of Regulation. Cambridge, S. 225-244.

Reif, Wolfgang (2010): Elitestudiengang Softwaretechnik. Vgl.: https://www.elitenetzwerk.bayern.de/ 61.0.html [Zugriff am 1.1.2010].

Reisz, Robert D. / Manfred Stock (2007 a): Inklusion in Hochschulen. Beteiligung an der Hochschulbildung und gesellschaftliche Entwicklung in Europa und in den USA (1950-2000). Bonn.

Reisz, Robert D. / Manfred Stock (2007b): Theorie der Weltgesellschaft und statistische Modelle im soziologischen Neoinstitutionalismus, in: Zeitschrift für Soziologie 36, S. 82-99. 
Schneevoigt, Ihno (2004): Eliteausbildung aus der Sicht der Wirtschaft, in: Beiträge zur Hochschulforschung 26 , H. 3, S. 46-53.

Schoefer, Evan / John W. Meyer (2005): The Worldwide Expansion of Higher Education in the Twentieth Century, in: American Sociological Review 70, S. 898-920.

Seib, Marion (2004): Neue Wege der Elitenförderung. Das Netzwerk der Exzellenz Deutschland und das Elitennetzwerk Bayern: Statement zu den politischen Vorgaben, in: Politische Studien, Heft 398, 55 Jg., S. 40-48.

Stichweh, Rudolf (2007): Die Universität in der Wissensgesellschaft: Wissensbegriffe und Umweltbeziehungen der modernen Universität. Vgl.: http://www.unilu.ch/files/die-universitaet-in-der-wissensgesellschaft.pdf [Zugriff 1.3.2009].

Stock, Manfred (2004): Steuerung als Fiktion. Anmerkungen zur Implementierung der neuen Steuerungskonzepte an Hochschulen aus organisationssoziologischer Sicht, in: Die Hochschule. Journal für Wissenschaft und Bildung 13, H. 1, S. 30-48.

Stock, Manfred (2005): Arbeiter, Unternehmer, Professioneller. Zur sozialen Konstruktion von Beschäftigung in der Moderne. Wiesbaden.

Studienkonzept (2007): Studienkonzept für das „Honors“-Bachelor-Modul an der Wirtschaftswissenschaftlichen Fakultät der Universität Regensburg. Regensburg 2007. Vgl.: http://132.199.124.212/honors/images/stories/struktur/Konzept-Honors-Bachelor.pdf [Zugriff am 1.2.2010].

Titze, Hartmut (1999): Zur Professionalisierung des höheren Lehramtes in der modernen Gesellschaft, in: Hans J. Apel / Klaus-Peter Horn / Peter Lundgreen (Hrsg.), Professionalisierung pädagogischer Berufe im historischen Prozess. Bad Heilbrunn. Vgl.: http://www.quakri.de/2_forschung/2c_publikationen/ Texte/1999b\%20Zur_Professionalisierung_des_h_heren_Lehramts_in_der_mod.pdf [Zugriff am 1.1.2010].

Trow, Martin (1972): The Expansion and Transformation of Higher Education, in: International Review of Education 18, S. 61-84.

Weber, Max (1988): Der Sozialismus, in: Max Weber, Gesammelte Aufsätze zur Soziologie und Sozialpolitik. Tübingen, S. 492-518.

PD Dr. Manfred Stock Martin-Luther-Universität Halle-Wittenberg Institut für Hochschulforschung Collegienstr. 62 06886 Wittenberg manfred.stock@hof.uni-halle.de 